\title{
Thermodynamic phase diagram of static charge order in underdoped $\mathrm{YBa}_{2} \mathrm{Cu}_{3} \mathrm{O}_{y}$
}

\author{
${\text { David LeBoeuf }{ }^{1 \star} \text {, S. Krämer }{ }^{2} \text {, W. N. Hardy }{ }^{3,4} \text {, Ruixing Liang }{ }^{3,4}, \text { D. A. Bonn }}^{3,4}$ and Cyril Proust ${ }^{1,4 \star}$
}

\begin{abstract}
The interplay between superconductivity and any other competing order is an essential part of the long-standing debate on the origin of high-temperature superconductivity in cuprate materials ${ }^{\mathbf{1 , 2}}$. Akin to the situation in the heavy fermions, organic superconductors and pnictides, it has been proposed that the pairing mechanism in the cuprates comes from fluctuations of a nearby quantum phase transition ${ }^{3}$. Recent evidence for charge modulation ${ }^{4}$ and its associated fluctuations ${ }^{5-7}$ in the pseudogap phase of $\mathrm{YBa}_{2} \mathrm{Cu}_{3} \mathrm{O}_{y}$ makes charge order a likely candidate for a competing order. However, a thermodynamic signature of the charge-ordering phase transition is still lacking. Moreover, whether the charge modulation is uniaxial or biaxial remains controversial. Here we address both issues by measuring sound velocities in $\mathrm{YBa}_{2} \mathrm{Cu}_{3} \mathrm{O}_{6.55}$ in high magnetic fields. We provide the first thermodynamic signature of the competing charge-order phase transition in $\mathrm{YBa}_{2} \mathrm{Cu}_{3} \mathrm{O}_{y}$ and construct a field-temperature phase diagram. The comparison of different acoustic modes indicates that the charge modulation is biaxial, which differs from a uniaxial stripe charge order.
\end{abstract}

In most La-based cuprate superconductors, static order of both spin and charge (so-called stripe order) has been unambiguously identified by spectroscopic and thermodynamic probes ${ }^{1,2}$. At low temperature, magnetic fields weaken superconductivity and at the same time reinforce the magnitude of such orders ${ }^{8-10}$. As the superconducting transition temperature $\left(T_{\mathrm{c}}\right)$ in the Labased materials is substantially lower than in other cuprate materials, it has been argued that stripe order is detrimental to high-temperature superconductivity. In underdoped $\mathrm{YBa}_{2} \mathrm{Cu}_{3} \mathrm{O}_{y}$ (YBCO), there is now compelling evidence of competing order even though $T_{\mathrm{c}}=94 \mathrm{~K}$ at optimal doping. The discovery of quantum oscillations ${ }^{11}$ combined with the negative Hall ${ }^{12}$ and Seebeck ${ }^{13}$ coefficients at low temperature has demonstrated that the Fermi surface of underdoped YBCO undergoes a reconstruction at low temperature and consists of at least one electron pocket. A comparative study of thermoelectric transport in underdoped YBCO and in $\mathrm{La}_{1.8-x} \mathrm{Eu}_{0.2} \mathrm{Sr}_{x} \mathrm{CuO}_{4}$ (a cuprate in which stripe order is well established) has been interpreted as charge stripe order causing reconstruction of the Fermi surface at low temperature ${ }^{14}$. High-field nuclear magnetic resonance (NMR) measurements have revealed that the translational symmetry of the $\mathrm{CuO}_{2}$ planes in $\mathrm{YBCO}$ is broken by the emergence of a modulation of the charge density at low temperature ${ }^{4}$. In addition, NMR measurements show that the modulation is observed above a threshold magnetic field and suggest that charge order is most likely uniaxial ${ }^{4}$. In zero field, long-range charge fluctuations in YBCO were recently observed with resonant soft X-ray scattering (RSXS) up to $150 \mathrm{~K}$ and $160 \mathrm{~K}$ for $p=$ 0.11 (ref. 5) and $p=0.133$ (ref. 6), respectively, whereas hard $\mathrm{X}$-ray scattering experiments suggest that a charge order develops below $135 \mathrm{~K}$ for $p=0.12$ (ref. 7). All measurements identify charge fluctuations at two wave vectors corresponding to an incommensurate periodicity of approximately 3.2 lattice units. The identification of a thermodynamic phase transition is thus important to determine where long-range charge order exists in the phase diagram and particularly whether static order occurs only in high magnetic fields.

Here we report sound velocity measurements, a thermodynamic probe, in magnetic fields large enough to suppress superconductivity. The sound velocity is defined as $v_{\mathrm{s}}=\sqrt{c_{i j} / \rho}$, where $\rho$ is the density of the material, $c_{i j}=\partial^{2} F / \partial \varepsilon_{i} \partial \varepsilon_{j}$ (ref. 15), $F$ is the free energy and $\varepsilon_{i}$ is the strain along direction $i$ (in the contracted Voigt notation). Changes in the elastic constants $c_{i j}$ are expected whenever a strain-dependent phase transition occurs. Owing to their high sensitivity, sound velocity measurements are a powerful probe for detecting such phase transitions, in particular charge ordering in strongly correlated electron systems ${ }^{16}$.

We have measured several elastic constants (see Supplementary Table S1 for the description of the elastic modes) in high magnetic fields in an underdoped $\mathrm{YBCO}_{6.55}$ sample with $T_{\mathrm{c}}=60.7 \mathrm{~K}$ corresponding to a hole doping $p=0.108$ (ref. 17). Figure $1 \mathrm{a}, \mathrm{b}$ shows the field dependence of the relative variation of the sound velocity $\Delta v_{\mathrm{s}} / v_{\mathrm{s}}$ corresponding to the $c_{11}$ mode, at different temperatures. At $T=4.2 \mathrm{~K}$, the softening of the elastic constant at the vortex lattice melting field $B_{\mathrm{m}} \approx 20 \mathrm{~T}$ corresponds to the firstorder melting transition from a vortex lattice to a vortex liquid ${ }^{18,19}$ (see Supplementary Information for more details). At $T=29.5 \mathrm{~K}$, this anomaly shifts to lower field $\left(B_{\mathrm{m}} \approx 5 \mathrm{~T}\right)$ and because the pinning potential becomes less effective, the magnitude of the change of $c_{11}$ at the melting transition becomes smaller ${ }^{20}$. At $T=29.5 \mathrm{~K}$ and above $B_{\mathrm{m}}$, a sudden increase of the elastic constant can clearly be resolved at $B_{\mathrm{co}}=18 \mathrm{~T}$, which corresponds to a thermodynamic signature of a phase transition. Whereas $B_{\mathrm{co}}$ is almost temperature independent at low temperature, it increases rapidly between 35 and $50 \mathrm{~K}$ (see red arrows in Fig. 1b). For $T \geq 50 \mathrm{~K}$, no change of $c_{11}$ can be resolved up to the highest field. Owing to the difference in the temperature dependence of $B_{\mathrm{m}}$ and $B_{\mathrm{co}}$, the phase transition at $B_{\mathrm{co}}$ cannot originate from vortices. Figure 2 shows the phase diagram in which both $B_{\mathrm{m}}$ and $B_{\mathrm{co}}$ deduced from sound velocity measurements are plotted as a function of temperature. The identification of this

\footnotetext{
${ }^{1}$ Laboratoire National des Champs Magnétiques Intenses, UPR 3228, (CNRS-INSA-UJF-UPS), Toulouse 31400, France, ${ }^{2}$ Laboratoire National des Champs Magnétiques Intenses, UPR 3228, (CNRS-INSA-UJF-UPS), Grenoble 38042, France, ${ }^{3}$ Department of Physics and Astronomy, University of British Columbia, Vancouver V6T 1Z1, Canada, ${ }^{4}$ Canadian Institute for Advanced Research, Toronto M5G 1Z8, Canada. *e-mail: david.leboeuf@Incmi.cnrs.fr; cyril.proust@Incmi.cnrs.fr.
} 
a

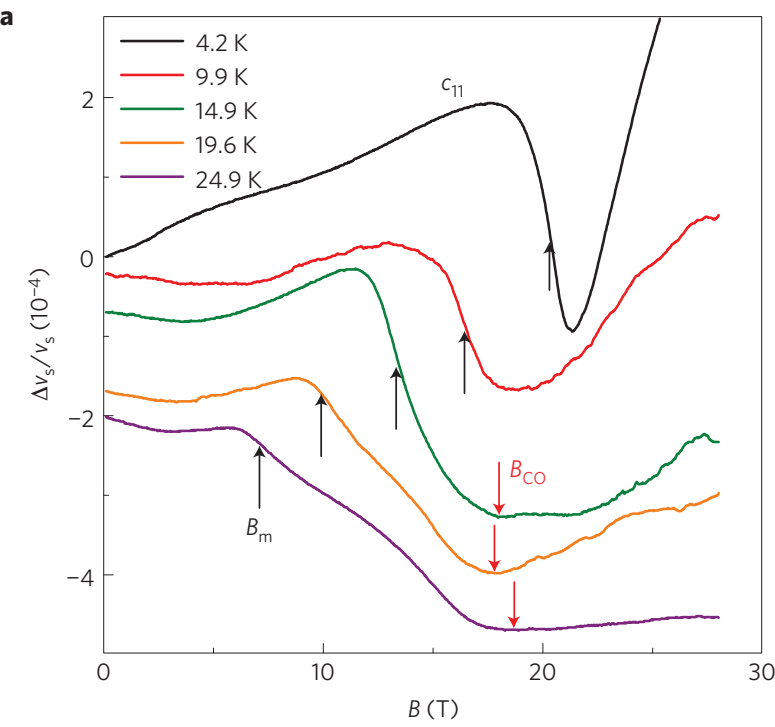

b

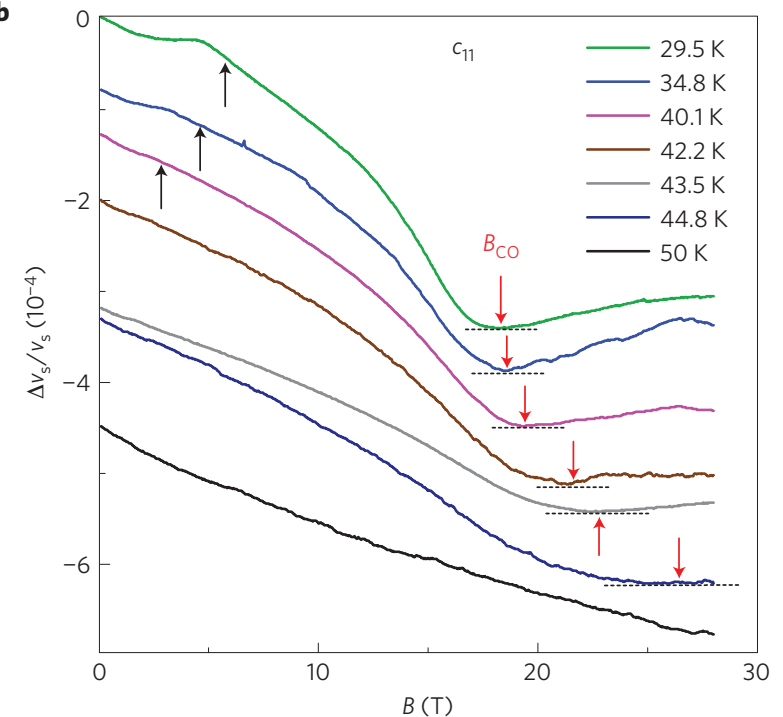

Figure 1 | Field dependence of the sound velocity in underdoped $\mathrm{YBa}_{2} \mathrm{Cu}_{3} \mathrm{O}_{\mathbf{y}} \mathbf{a} \mathbf{a}, \mathbf{b}$, Field dependence of the longitudinal mode $c_{11}$ (propagation $q$ and polarization $u$ of the sound wave along $a$ axis) in underdoped YBCO ( $p=0.108)$ at different temperatures from $T=4.2 \mathrm{~K}$ to $T=24.9 \mathrm{~K}(\mathbf{a})$, and from $T=29.5 \mathrm{~K}$ to $T=50 \mathrm{~K}(\mathbf{b})$. The curves are shifted for clarity. The measurements were performed in static magnetic field up to $28 \mathrm{~T}$. Black arrows indicate the field $B_{m}$ corresponding to the vortex lattice melting. At low temperature, the loss of the vortex lattice compression modulus can be estimated and is in agreement with previous studies (see Supplementary Information). For $T>40 \mathrm{~K}, B_{\mathrm{m}}$ cannot be resolved. Red arrows indicate the field $B_{\text {co }}$ where the charge-order phase transition occurs. This transition is not related to vortex physics because it is also seen in acoustic modes $\mathrm{C}_{44}$ and $\mathrm{c}_{55}$ (Fig. 3 and Supplementary Fig. S3), which are insensitive to the flux line lattice because those modes involve atomic motions parallel to the vortex flux lines $(u\|H\| c)$.

phase stabilized by the magnetic field above $B_{\text {co }}$ is straightforward. High-field NMR measurements in YBCO at similar doping have shown that charge order develops above a threshold field $B_{\mathrm{co}}>15 \mathrm{~T}$ and below $T_{\text {co }}^{\mathrm{RMN}}=50 \pm 10 \mathrm{~K}$ (ref. 4). Given the similar field and temperature scales, it is natural to attribute the anomaly seen in the elastic constant at $B_{\text {co }}$ to the thermodynamic transition towards the static charge order.

The phase diagram in Fig. 2 shares common features with the theoretical phase diagram of superconductivity in competition

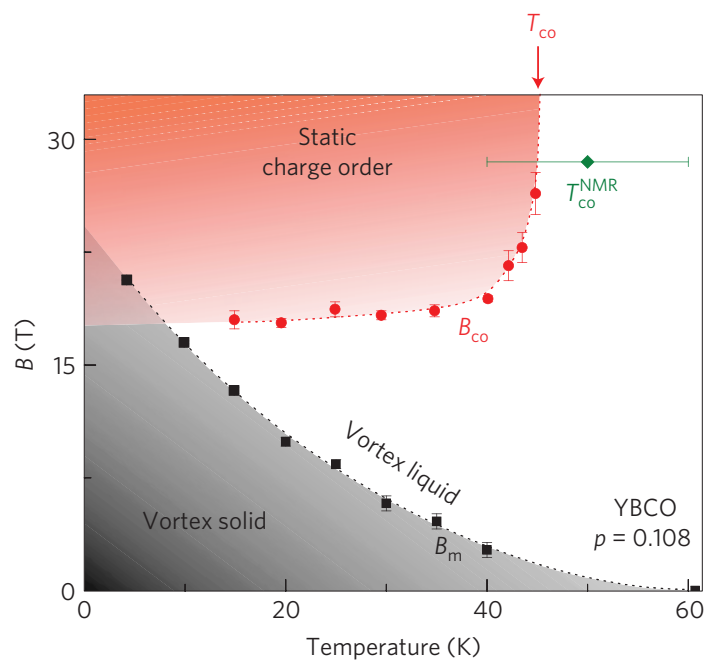

Figure 2 | Thermodynamic phase diagram. Magnetic field-temperature phase diagram of underdoped YBCO $(p=0.108)$ obtained from the anomalies seen in the elastic constant $c_{11}$ (Fig. 1). Black squares indicate the transition from a vortex lattice to a vortex liquid at $B_{\mathrm{m}}$, which cannot be resolved above $40 \mathrm{~K}$. Red circles correspond to the phase transition towards static charge order at $B_{\mathrm{co}}$, as observed in $c_{11}$. The error bars on the field scale $B_{\mathrm{m}}\left(B_{\mathrm{co}}\right)$ correspond to the width of the transition in the derivative (raw data) of $C_{11}(B)$. The charge-order transition is almost temperature independent up to $\approx 40 \mathrm{~K}$. Above $40 \mathrm{~K}$ the field scale $B_{\mathrm{co}}$ at which charge order sets in rises. In the Supplementary Information, we argue that the overall behaviour of the charge-order phase boundary in this $B-T$ diagram is consistent with a theoretical model of superconductivity in competition with a density-wave state ${ }^{21}$. The green diamond is the temperature $T_{\mathrm{co}}^{\mathrm{NMR}}=50 \pm 10 \mathrm{~K}$ at which NMR experiments detect the onset of a charge modulation at a field $B=28.5 \mathrm{~T}$ in YBCO at doping $p=0.11$ (ref. 4). Within the error bars, this onset temperature agrees with our findings. Dashed lines are guides to the eye.

with a density-wave order ${ }^{21}$ (see discussion in the Supplementary Information). For $T$ below $40 \mathrm{~K}$ or so, static charge order sets in only above a threshold field of $18 \mathrm{~T}$, akin to the situation in $\mathrm{La}_{2-x} \mathrm{Sr}_{x} \mathrm{CuO}_{4}(x=0.145)$ in which a magnetic field is necessary to destabilize superconductivity and to drive the system to a magnetically ordered state ${ }^{9}$. Close to the onset temperature of static charge order, $T_{\text {co }}$, the threshold field $B_{\text {co }}$ sharply increases and the phase boundary tends to become vertical. This is in agreement with the theoretical phase of competing order with superconductivity that predicts that superconducting fluctuations have no significant effect on charge order in this part of the phase diagram.

We now turn to the analysis of the symmetry of the charge modulation. In the framework of the Landau theory of phase transitions, an anomaly in the elastic constant occurs at a phase transition only if a coupling in the free energy $F_{\mathrm{c}}=g_{m n} Q^{m} \varepsilon^{n}$ (where $m$ and $n$ are integers and $g_{m n}$ is a coupling constant) between the order parameter $Q$ and the strain $\varepsilon$ is symmetry allowed, that is, only if $Q^{m}$ and $\varepsilon^{n}$ transform according to the same irreducible representation $^{22}$. In Fig. 3 we compare the field dependence at $T=20 \mathrm{~K}$ of four different modes $c_{11}, c_{44}, c_{55}$ and $c_{66}$ that display an anomaly at $B_{\mathrm{co}}$. To explain the presence of such coupling for all these modes, we rely on group theory arguments. YBCO is an orthorhombic system (point group $D_{2 h}$ ), and given the even character of the strains we have only to consider the character table of point group $D_{2}$ shown in Table 1 .

To represent the different symmetric charge modulations that transform according to each irreducible representation of the point group $\mathrm{D}_{2}$ and to determine to which acoustic mode they couple, we 


\section{Table 1 | Character table of point group $D_{2}$.}

\begin{tabular}{lcrrrll} 
Irr. rep. & $\boldsymbol{E}$ & $\mathbf{C}_{\mathbf{2}}^{\mathbf{z}}$ & $\boldsymbol{C}_{\mathbf{2}}^{\mathbf{y}}$ & $\boldsymbol{C}_{\mathbf{2}}^{\mathbf{x}}$ & $\begin{array}{l}\text { Basis } \\
\text { functions }\end{array}$ & $\begin{array}{l}\text { Symmetric } \\
\text { strains }\end{array}$ \\
\hline$A_{1 g}$ & 1 & 1 & 1 & 1 & $x^{2}, y^{2}, z^{2}$ & Volume strains: $\varepsilon_{1}$ \\
$B_{1 g}$ & 1 & 1 & -1 & -1 & $z, x y$ & $\varepsilon_{6}$ \\
$B_{2 g}$ & 1 & -1 & 1 & -1 & $y, x z$ & $\varepsilon_{5}$ \\
$B_{3 g}$ & 1 & -1 & -1 & 1 & $x, y z$ & $\varepsilon_{4}$
\end{tabular}

Character table, basis functions and transformation properties of the strains for the point group $D_{2}$ appropriate for $Y B C O$. The $z, y$ and $x$ axes correspond to the crystalline $c, b$ and $a$ axes of the YBCO structure, respectively. Irr. rep., irreducible representation.

will follow the procedure of ref. 16 and use the projection operator in the basis defined by the four $\mathrm{Cu}$ atoms at the corner of the $\mathrm{CuO}_{2}$ plane (see Supplementary Information). This simplification is justified because the charge order is an intrinsic property of the $\mathrm{CuO}_{2}$ plane ${ }^{4,6}$. The four kinds of symmetric charge distribution allowed in this basis are shown in Supplementary Fig. S5. In Fig. 4, we illustrate them with concrete charge modulation patterns in the $\mathrm{CuO}_{2}$ plane with an arbitrary periodicity. A uniaxial charge modulation along the $a$ axis should induce an anomaly in $c_{44}$ but not in $c_{55}$. The very similar field dependence of $c_{44}$ and $c_{55}$ around $B_{\text {co }}$ (Fig. 3) indicates a biaxial charge modulation both along the $a$ axis $\left(Q_{a}\right)$ and along the $b$ axis $\left(Q_{b}\right)$. As the irreducible representation $B_{1 g}=B_{2 g} \otimes B_{3 g}$ and $\varepsilon_{6}$ transforms according to $B_{1}$, a further coupling $\beta_{a b} Q_{a} Q_{b} \varepsilon_{6}$ explains the anomaly seen in $c_{66}$. A similar coupling exists for $c_{11}$ because $A_{1 g}=B_{i g} \otimes B_{i g}(i=1,2$ or 3$)$.

As a thermodynamic bulk probe with a typical energy scale below $1 \mu \mathrm{eV}$, sound velocity measurements establish that static charge order sets in below $T_{\mathrm{co}} \approx 45 \mathrm{~K}$ in magnetic fields above $B_{\mathrm{co}}=18 \mathrm{~T}$ in $\mathrm{YBCO}$ at a doping level $p=0.108$. For a nearby doping $p=0.11$ (non-ortho-II ordered sample), zerofield RSXS measurements show that charge fluctuations appear at $T=150 \mathrm{~K}$ (ref. 5). The fluctuating character of the charge order at high temperature is consistent with recent resonant ultrasonic measurements in YBCO at similar doping level. Whereas anomalies in the sound velocity are detected at the pseudogap temperature, $T^{*} \approx 240 \mathrm{~K}$, and at the superconducting transition temperature $T_{c}$, no further phase transition is observed in zero field ${ }^{23}$. An important aspect of these fluctuations is their biaxial character inferred from both RSXS (refs 5,6) and X-ray ${ }^{7}$ measurements. However, those techniques cannot determine whether there is a single charge density wave (CDW) with biaxial modulation or whether there are domains of two uniaxial CDWs. From sound velocity measurements, the latter scenario seems unlikely because for the anomaly in $c_{66}$ to exist, charge modulations along the two directions should in principle coexist within the same sample volume. The biaxial character of the CDW observed in our study and in X-ray measurements supports the scenario in which the static charge ordering occurring at low temperature and finite magnetic field is a consequence of the freezing of the charge fluctuations observed in zero field at high temperature. A biaxial CDW seems to differ from the conclusion drawn from NMR measurements, which have been interpreted as a uniaxial modulation of the charge density along the $a$ axis ${ }^{4}$. However, an extra charge modulation along the $b$ axis, as reported here, is not excluded from the NMR spectra. The biaxial nature of the CDW differs from the phenomenology of stripe order, which corresponds to a uniaxial charge order, as observed in La-based cuprates $^{1,2}$. This is further supported by the absence of spin order down to the lowest temperature at this doping level as inferred from NMR measurements ${ }^{4}$. It raises the questions of the origin of the $T_{\mathrm{c}}$ anomaly close to $p=0.12$ in underdoped a

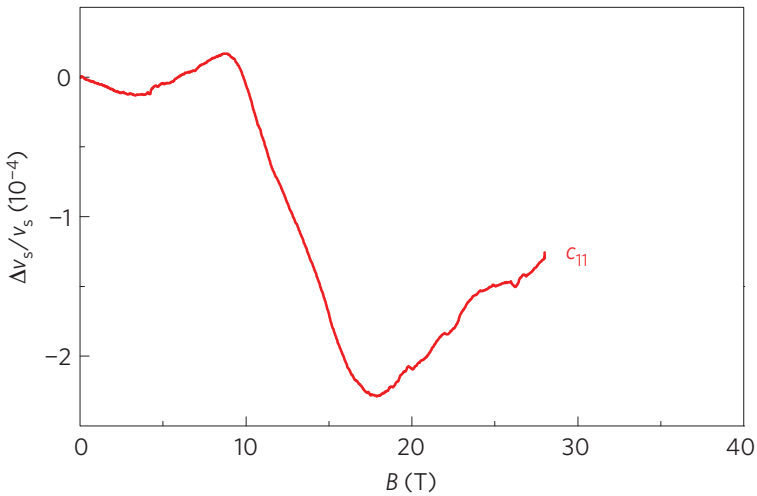

b
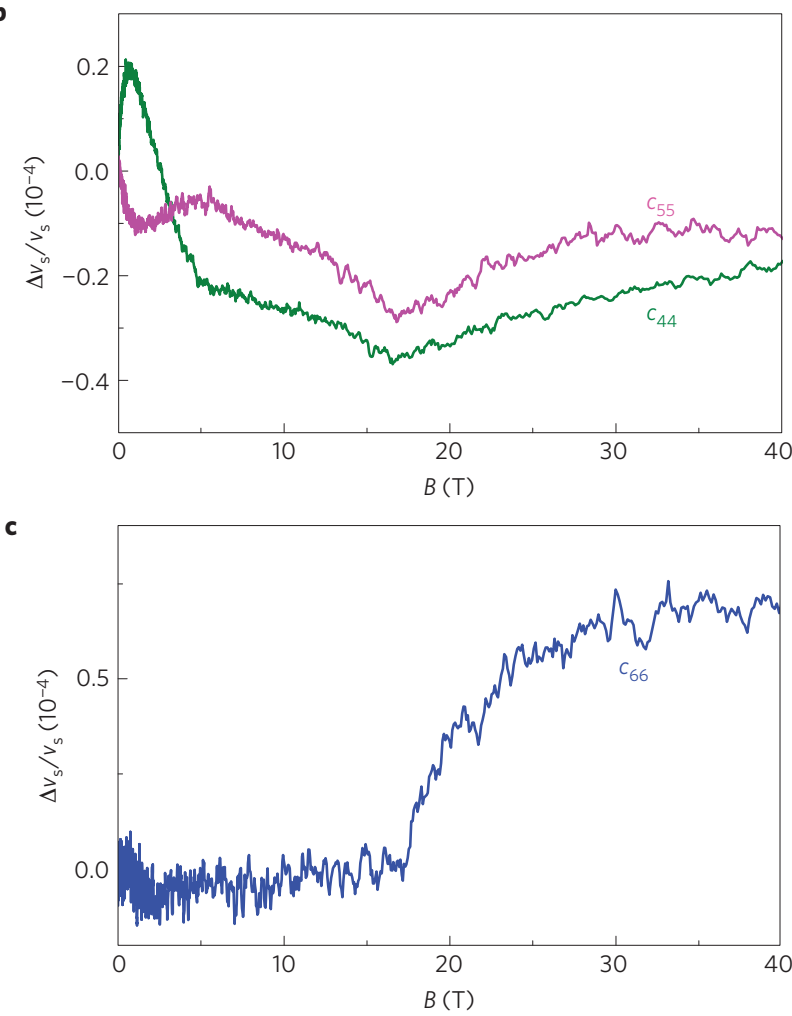

Figure 3 | Charge-order transition seen in different modes. Field dependence of different elastic constants of YBCO $(p=0.108)$ at $T=20 \mathrm{~K}$. a, Longitudinal mode $c_{11} . \mathbf{b}, c_{44}(q\|b, u\| c)$ in green and $c_{55}(q\|a, u\| c)$ in magenta. c, $c_{66}(q\|b, u\| a) . c_{44}$ and $c_{55}$ have similar amplitude and field dependence at the transition $B_{\mathrm{co}}$ towards the charge order, indicating the biaxial character of the charge-density modulation. The anomalies seen in $c_{66}$ and $c_{11}$ are larger than in $c_{44}$ and $c_{55}$. This is probably due to a greater dependence of charge order with respect to in-plane strains than out-of-plane strains.

YBCO and of the peculiar behaviour of the transport properties at high fields around this doping level ${ }^{24}$. These questions and the interplay between biaxial CDW and high-temperature superconductivity call for further investigations. The biaxial character of the charge order has another important consequence, namely on the topology of the reconstructed Fermi surface. In the absence of spin order, as inferred from NMR (ref. 4), uniaxial order by itself cannot produce electron pockets in the reconstructed Fermi surface ${ }^{25}$, unless one assumes that the Fermi surface at high temperature already breaks $C_{4}$ symmetry $^{26}$. On the other hand, no particular assumption is needed to produce electron pockets when the Fermi surface is folded along two perpendicular directions ${ }^{27}$. 


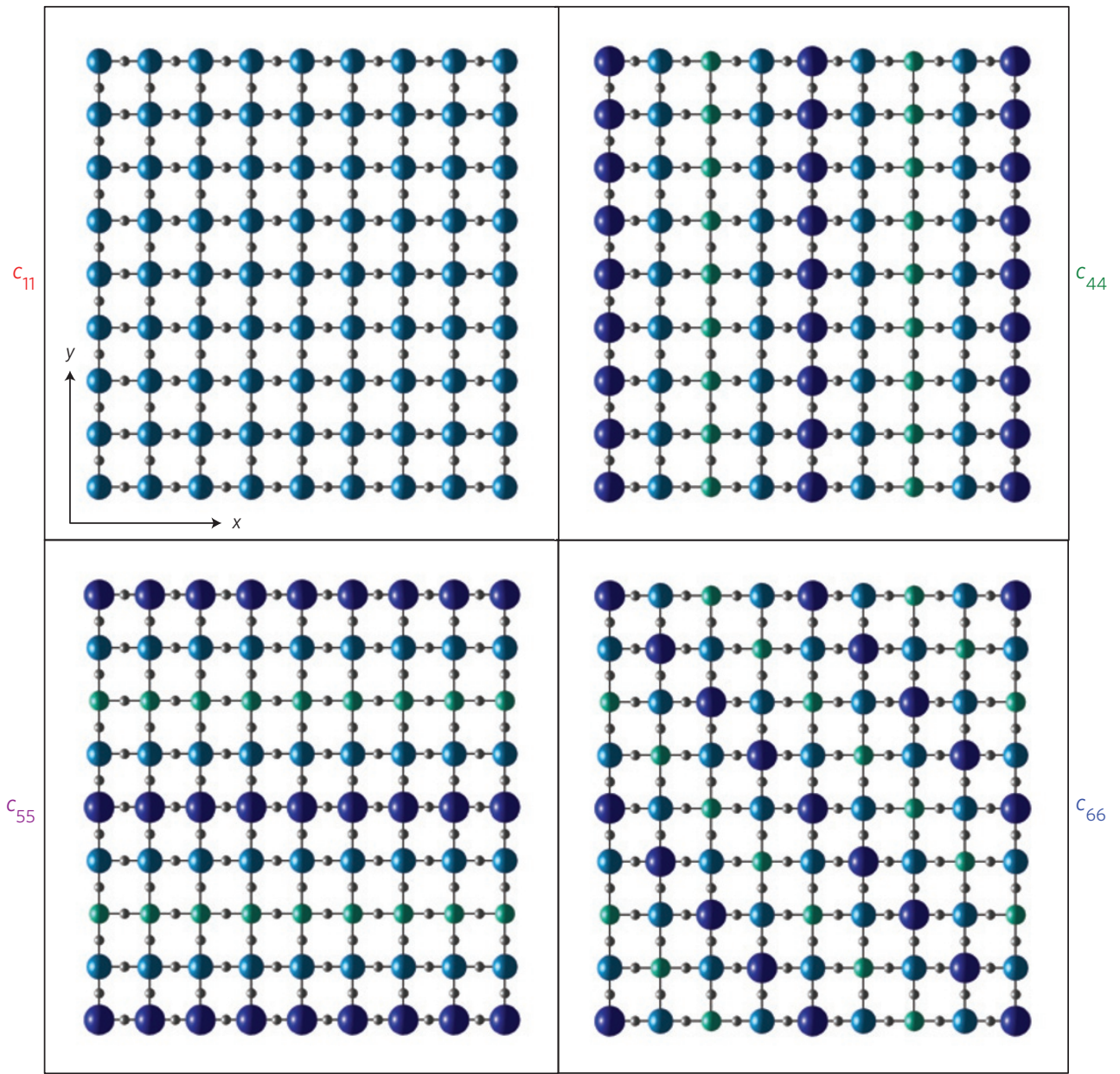

Figure 4 | Charge distribution in the $\mathrm{CuO}_{2}$ plane. Sketches of the different charge modulation patterns in the $\mathrm{CuO}_{2}$ plane coupled with the measured elastic constants. Blue and green spheres represent $\mathrm{Cu}$ atoms with different charge density. Grey spheres represent $\mathrm{O}$ atoms. The periodicity of the modulation is arbitrary. We assume that charge modulation takes place on the $\mathrm{Cu}$ atom, but including modulation of the charge on the $\mathrm{O}$ atoms does not change the conclusion. If the charge distribution is uniform, anomalies would be seen only in $\mathrm{c}_{11}$. A uniaxial charge order with a modulation along the $a(b)$ axis couples linearly with $c_{44}\left(c_{55}\right)$. The fact that both $c_{44}$ and $c_{55}$ show an anomaly at $B_{c 0}$ indicates that the charge modulation is biaxial.

Received 14 September 2012; accepted 11 November 2012; published online 23 December 2012

\section{References}

1. Kivelson, S. A. et al. How to detect fluctuating stripes in the high-temperature superconductors. Rev. Mod. Phys. 75, 1201-1241 (2003).

2. Vojta, M. Lattice symmetry breaking in cuprate superconductors: Stripes, nematics, and superconductivity. Adv. Phys. 58, 699-820 (2009).

3. Taillefer, L. Scattering and pairing in cuprate superconductors. Annu. Rev. Condens. Matter Phys. 1, 51-70 (2010).

4. $\mathrm{Wu}, \mathrm{T}$. et al. Magnetic-field-induced charge-stripe order in the high-temperature superconductor $\mathrm{YBa}_{2} \mathrm{Cu}_{3} \mathrm{O}_{y}$. Nature 477, 191-194 (2011).

5. Ghiringhelli, G. et al. Long-range incommensurate charge fluctuations in (Y, Nd) $\mathrm{Ba}_{2} \mathrm{Cu}_{3} \mathrm{O}_{6+x}$. Science 337, 821-825 (2012).

6. Achkar, A. J. et al. Distinct charge orders in the planes and chains of ortho-III ordered $\mathrm{YBa}_{2} \mathrm{Cu}_{3} \mathrm{O}_{6+\delta}$ superconductors identified by resonant X-ray scattering Phys. Rev. Lett. 109, 167001 (2012).

7. Chang, J. et al. Direct observation of competition between superconductivity and charge density wave order in $\mathrm{YBa}_{2} \mathrm{Cu}_{3} \mathrm{O}_{y}$. Nature Phys 8, 871-876 (2012).

8. Lake, B. et al. Antiferromagnetic order induced by an applied magnetic field in a high-temperature superconductor. Nature 415, 299-302 (2002).

9. Chang, J. et al. Tuning competing orders in $\mathrm{La}_{2-x} \mathrm{Sr}_{x} \mathrm{CuO}_{4}$ cuprate superconductors by the application of an external magnetic field. Phys. Rev. B 78, 104525 (2008).

10. Wen, J. et al. Uniaxial linear resistivity of superconducting $\mathrm{La}_{1.905} \mathrm{Ba}_{0.095} \mathrm{CuO}_{4}$ induced by an external magnetic field. Phys. Rev. B 85, 134513 (2012).
11. Doiron-Leyraud, N. et al. Quantum oscillations and the Fermi surface in an underdoped high- $T_{c}$ superconductor. Nature 447, 565-568 (2007).

12. LeBoeuf, D. et al. Electron pockets in the Fermi surface of hole-doped high- $T_{\mathrm{c}}$ superconductors. Nature 450, 533-536 (2007).

13. Chang, J. et al. Nernst and Seebeck coefficients of the cuprate superconductor $\mathrm{YBa}_{2} \mathrm{Cu}_{3} \mathrm{O}_{6.67}$ : A study of Fermi surface reconstruction. Phys. Rev. Lett. 104, 057005 (2010).

14. Laliberté, F. et al. Fermi-surface reconstruction by stripe order in cuprate superconductors. Nature Commun. 2, 432 (2011).

15. Lüthi, B. Springer Series for Solid-State Sciences Vol. 148 (Springer, 2005).

16. Goto, T \& Lüthi, B. Charge ordering, charge fluctuations and lattice effects in strongly correlated electron systems. Adv. Phys. 52, 67-118 (2003).

17. Liang, R. et al. Evaluation of $\mathrm{CuO}_{2}$ plane hole doping in $\mathrm{YBa}_{2} \mathrm{Cu}_{3} \mathrm{O}_{6+x}$ single crystals. Phys. Rev. B 73, 180505 (2006).

18. Liang, R. et al. Discontinuity of reversible magnetization in untwinned YBCO single crystals at the first order vortex melting transition. Phys. Rev. Lett. 76, 835-838 (1996).

19. Ramshaw, B. et al. Vortex lattice melting and $H_{c 2}$ in underdoped $\mathrm{YBa}_{2} \mathrm{Cu}_{3} \mathrm{O}_{y}$. Phys. Rev. B 86, 174501 (2012).

20. Pankert, J. et al. Ultrasonic attenuation by the vortex lattice of high- $T_{\mathrm{c}}$ superconductors. Phys. Rev. Lett. 65, 3052-3055 (1990).

21. Moon, E. G. \& Sachdev, S. Competition between spin density wave order and superconductivity in the underdoped cuprates. Phys. Rev. B 80, 035117 (2009).

22. Rehwald, W. The study of structural phase transitions by means of ultrasonic experiments. Adv. Phys. 22, 721-755 (1973).

23. Shekhter, A. Ultrasonic signatures at the superconducting and the pseudogap phase boundaries in YBCO cuprates. Preprint at http://arXiv.org/abs/1208.5810 (2012). 
24. LeBoeuf, D. et al. Lifshitz critical point in the cuprate superconductor $\mathrm{YBa}_{2} \mathrm{Cu}_{3} \mathrm{O}_{y}$ from high-field Hall effect measurements. Phys. Rev. B 83, 054506 (2011).

25. Millis, A. J. \& Norman, M. R. Antiphase stripe order as the origin of electron pockets observed in 1/8-hole-doped cuprates. Phys. Rev. B 76, 220503 (2007).

26. Yao, H. et al. Fermi-surface reconstruction in a smectic phase of a high-temperature superconductor. Phys. Rev. B 84, 012507 (2011).

27. Sebastian, S. et al. Quantum oscillations from nodal bilayer magnetic breakdown in the underdoped high temperature superconductor $\mathrm{YBa}_{2} \mathrm{Cu}_{3} \mathrm{O}_{6+x}$. Phys. Rev. Lett. 108, 196403 (2012).

\section{Acknowledgements}

We thank M-H. Julien, S. Kivelson, B. Lüthi, R. Ramazashvili, G. Rikken, L. Taillefer, B. Vignolle, M. Vojta and S. Zherlitsyn for useful discussions. We acknowledge experimental support from A. Mari, D. Rickel and the LNCMI staff. Research support was provided by the French ANR SUPERFIELD, Euromagnet II, the Canadian Institute for Advanced Research and the Natural Science and Engineering Research Council.

\section{Author contributions}

D.L. and C.P. performed the high-field measurements. S.K. provided instrumental support for the d.c. field measurements. W.N.H., R.L. and D.A.B. prepared the samples (crystal growth, annealing, de-twinning). D.L. analysed the data. D.L. and C.P. wrote the manuscript and C.P. supervised the project.

\section{Additional information}

Supplementary information is available in the online version of the paper. Reprints and permissions information is available online at www.nature.com/reprints.

Correspondence and requests for materials should be addressed to D.L. or C.P.

\section{Competing financial interests}

The authors declare no competing financial interests. 